\title{
Utilização de prototipagens em cirurgia e traumatologia bucomaxilofacial: relato de casos
}

\section{Use of prototyping in oral and maxillofacial surgery and traumatology: case reports}

\author{
Rafael Morawski" \\ Lucas Bozzetti Pigozzi" \\ Kiara Fabro* \\ Leonardo Tonietto** \\ Vinícius Salim Silveira ${ }^{* * *}$ \\ Thiago Calcagnotto ${ }^{* * *}$
}

\section{Resumo}

Objetivo: apresentar as possibilidades da utilização da prototipagem (biomodelos) em cirurgia e traumatologia bucomaxilofacial por meio do relato de casos clínicos. Relato de casos: o presente estudo abrange três situações clínicas, sendo o primeiro caso o de um paciente com osteorradionecrose associado à fratura patológica devido à evolução do quadro de osteólise, sendo realizada a ressecção do segmento e a adaptação da placa de reconstrução no biomodelo. O segundo caso relata a presença de um ameloblastoma multicístico, abrangendo a sínfise, a para-sínfise e o corpo da mandíbula, sendo utilizada a prototipagem para adaptação da placa cirúrgica de reconstrução. No último relato, a paciente foi diagnosticada com Tumor Epitelial Calcificante, desde a linha média da maxila até a fossa ptérigo-maxila do lado esquerdo. Por intermédio do uso de biomodelos, foi possível confeccionar uma prótese total implantossuportada que realizasse o vedamento do defeito ósseo. Considerações finais: é notável que o uso de biomodelos é auxiliar no planejamento e na execução cirúrgica, expandindo alternativas de tratamento que possibilitem a reabilitação do paciente com técnicas cirúrgicas mais eficazes, com menor morbidade e maior rapidez cirúrgica.

Palavras-chave: Impressão tridimensional. Cirurgia bucal. Patologia.

\section{Introdução}

A utilização de prototipagens (modelos impressos da estrutura óssea do paciente) na odontologia vem aumentando muito nos últimos anos devido à gama de vantagens oferecidas por esse recurso. Com a crescente busca por diagnósticos mais precisos e tratamentos menos agressivos, as prototipagens surgem como ferramentas auxiliares que ajudam a simplificar casos considerados de maior complexidade, minimizando assim o tempo cirúrgico e os longos períodos de morbidade pós-operatória ${ }^{1,2}$. Esses modelos são confeccionados a partir de exames de imagens tomográficas ou ressonâncias magnéticas no formato DICOM (Digital Imaging and Comunications in Medicine) e impressos com o sistema CAD (Computer Aided Design). Essa interação possibilita a criação de uma estrutura tridimensional que representa um modelo físico do paciente com estrutura anatômica óssea muito semelhante à natural, que, com a espessura milimétrica dos cortes tomográficos, determina a fidelidade do biomodelo impresso tridimensionalmente ${ }^{3,4}$. Para que seja possível a confecção desses modelos, existem duas técnicas: a subtrativa e a aditiva. No método subtrativo, o material é esculpido por meio de um bloco íntegro inicial. Já no aditivo, a máquina adiciona

\footnotetext{
Acadêmicos em Odontologia na Universidade Federal do Rio Grande do Sul.

Mestre em CTBMF pela Pontifícia Universidade Católica do Rio Grande do Sul. Professor do Curso de Especialização em CTBMF - Fatec Dental CEEO. * Doutor em CTBMF pela Pontifícia Universidade Católica do Rio Grande do Sul. Professor do Curso de Especialização em CTBMF - Fatec Dental CEEO.

${ }^{* * *}$ Mestre em CTBMF pela Universidade Federal do Rio Grande do Sul. Coordenador do Curso de Especialização em CTBMF - Fatec Dental CEEO.
} 
várias camadas do material, uma sobre a outra, de acordo com o formato desejado do modelo ${ }^{5}$.

Com o biomodelo em mãos, o cirurgião-dentista tem a possibilidade de utilização em consultório como ferramenta auxiliar de diagnóstico e método facilitador do tratamento. As principais áreas da odontologia que utilizam os biomodelos são a implantodontia, a cirurgia bucomaxilofacial e a prótese bucomaxilofacial. Quando se refere à implantodontia, essa tecnologia permite a visualização em $3 \mathrm{D}$ das estruturas ósseas do paciente, tornando o planejamento cirúrgico mais seguro e a execução mais rápida, com maior precisão operatória e melhor previsibilidade dos resultados ${ }^{6}$. Na cirurgia bucomaxilofacial, reduz-se o tempo cirúrgico quando o planejamento é realizado sobre o biomodelo do paciente, uma vez que há redução de danos aos tecidos, manutenção do contorno facial do paciente e manutenção da posição da articulação temporomandibular (ATM) sem causar alterações biodinâmicas no pós-cirúrgico ${ }^{7,8}$.

Em relação às próteses bucomaxilofaciais, como próteses auriculares, nasais e próteses obturadoras, os biomodelos representam uma ótima ferramenta auxiliar na fabricação de guias cirúrgicos para delimitação de extensas lesões patológicas, como em pacientes que apresentam lesões patológicas destrutivas como cistos e tumores odontogênicos. Porém, esse método utiliza uma tecnologia sofisticada e ainda recente, fato que tem algumas desvantagens, como o alto custo para confecção dessas prototipagens, a escassez de locais que realizam a impressão do biomodelo e poucos estudos que comprovem de maneira confiável a precisão da estrutura anatômica do paciente no biomodelo ${ }^{9}$.

Assim, o presente trabalho tem o objetivo de ilustrar e discutir, com uma revisão de literatura e a apresentação de três casos, as possibilidades da utilização de biomodelos em cirurgia e traumatologia bucomaxilofacial.

\section{Relato de casos}

\section{Caso clínico 1}

Paciente do gênero masculino, raça negra, 61 anos de idade e com histórico de realização de cirurgia e radioterapia em região de cabeça e pescoço em função do tratamento de câncer de laringe há três anos. Foi encaminhado para avaliação no serviço de Cirurgia e Traumatologia Bucomaxilofacial do Hospital Bom Pastor, em Igrejinha, RS, devido à presença de fístula extraoral na região de corpo mandibular à direita. $\mathrm{O}$ exame clínico evidenciou área de exposição óssea intrabucal nessa região e fístula ativa na região submandibular desse mesmo lado. O exame tomográfico confirmou diagnóstico clínico de osteoradionecrose associado à fratura patológica devido à evolução do quadro de osteólise.

A proposta de tratamento foi a ressecção do segmento comprometido até que se observasse margem óssea vital com prévia fixação de placa de reconstrução Sistema NeoOrtho 2.4 (NeoOrtho - JJGC Ind. e Com. de Materiais Dentário S/A, Curitiba, Brasil). O exame tomográfico (Figura 1-A) em formato DICOM permitiu a confecção de um biomodelo mandibular (Figura 1-B). Previamente à cirurgia, realizou-se a adaptação da placa de reconstrução no Sistema 2.4 sobre o biomodelo do paciente (Figura 1-B). Essa placa foi esterilizada em autoclave a $121^{\circ} \mathrm{C}$, com $1 \mathrm{~atm}$ de pressão, por 45 minutos. A cirurgia foi realizada por meio de acesso intrabucal com incisão linear sobre o rebordo alveolar, com extensão de ramo mandibular da direita até o ramo mandibular à esquerda. Após o descolamento mucoperiostal, a placa de reconstrução foi fixada (Figura 1-C) e, em seguida, com a utilização do sistema de corte Piezzo (Piezzo Surgery, NSK - Nakanishi International, Tóquio, Japão), o segmento ósseo mandibular comprometido foi removido (Figura 1-D). 

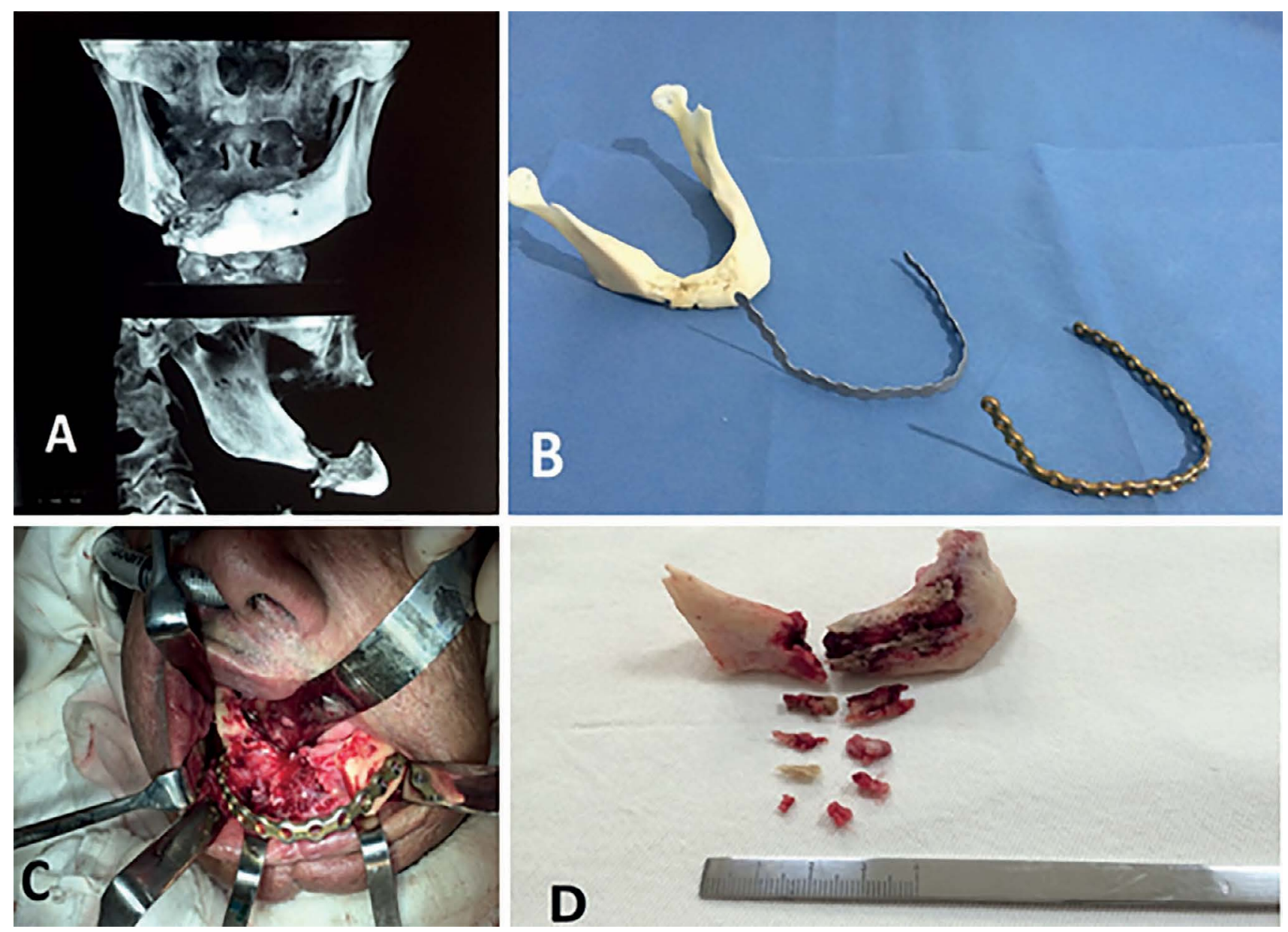

Figura 1 - Imagens pré e transoperatórias de ressecção de segmento ósseo mandibular devido à destruição por osteoradionecrose

A) Imagem tomográfica evidenciando fratura patológica devido à osteoradionecrose; B) Imagem do biomodelo utilizado para modelar e adaptar a placa de reconstrução previamente à cirurgia; C) Imagem transoperatória evidenciando placa adaptada e segmento ósseo afetado por osteorradrionecrose removido; D) segmento ósseo com osteoradionecrose removido.

Fonte: elaboração dos autores.

A utilização dessa técnica cirúrgica buscou a diminuição do tempo cirúrgico, uma vez que a placa de reconstrução foi modelada previamente à cirurgia, sendo adaptada sobre o biomodelo do paciente. Ainda, permitiu que o acesso cirúrgico fosse realizado de forma intrabucal, diminuindo a morbidade pós-operatória e os riscos de paralisias faciais quando comparado a um acesso cirúrgico extrabucal.

\section{Caso clínico 2}

Paciente do gênero masculino, raça branca, 47 anos de idade, encaminhado ao serviço de Cirurgia e Traumatologia Bucomaxilofacial do Hospital Bom Pastor, em Igrejinha, RS, para avaliação de lesão osteolítica na região anterior de mandíbula, com limites abrangendo sínfise, para-sínfise e corpo de mandíbula, com diagnóstico histopatológico de ameloblastoma multicístico. $\mathrm{O}$ exame tomográfico de imagem evidenciou uma lesão hipodensa com limites da região do dente 35 à região do dente 46 (Figura 2-A), com expansão e destruição de corticais vestibular e lingual. Ainda, a partir do exame de imagem, realizou-se a confecção de um biomodelo da mandíbula do paciente (Figura 2-B) e, a partir dos achados clínicos e imaginológicos, o plano de tratamento foi de realizar a fixação de uma placa de reconstrução bilateral (Sistema Look 2.4, Synthes, Jhonson \& Jhonson, Jhonson \& Jhonson do Brasil Ind. e Com. de Produtos de Saúde, São José dos Campos, SP, Brasil). Após a realização da fixação da placa foi realizada a remoção do segmento patológico com margem de segurança de $1,5 \mathrm{~cm}$ em cada extremo da lesão. $\mathrm{O}$ acesso cirúrgico foi intrabucal, realizado por meio de incisão linear sobre o rebordo alveolar com extensão de ramo mandibular à direita até o ramo mandibular à esquerda. Após o descolamento mucoperiostal, a placa de reconstrução foi fixada (Figura 2-C) e, em seguida, com a utilização de serra reciprocante, o segmento mandibular com a lesão foi removido.

A modelagem da placa sobre o biomodelo e a fixação dessa previamente à remoção da lesão permitiram o acesso cirúrgico por meio de incisão intrabucal, fato que diminuiu a morbidade pós-operatória, permitiu a manutenção do contorno facial do paciente (Figura 2-D) e manteve as cabeças da mandíbula em posição fisiológica adequada. 

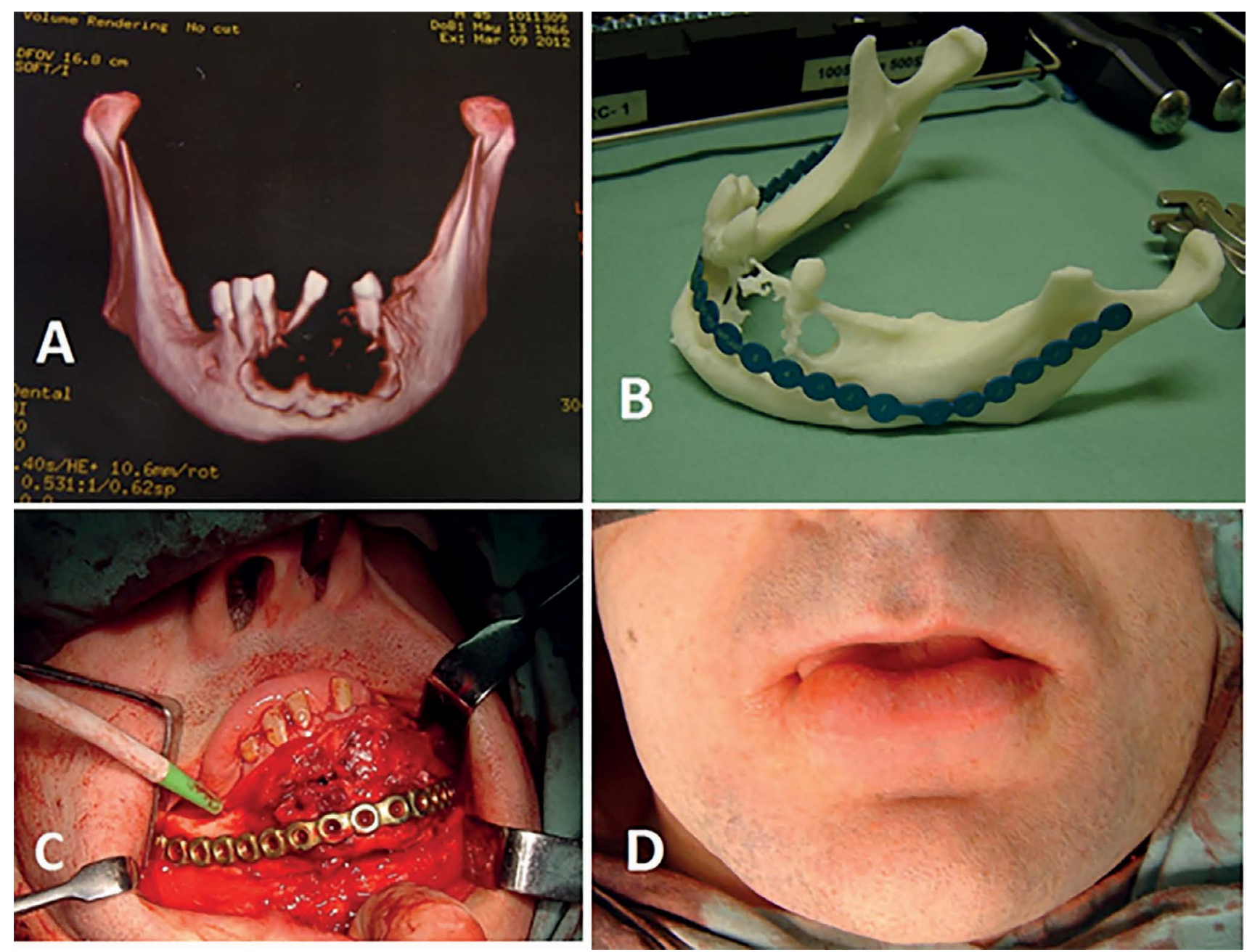

Figura 2 - Imagens pré e transoperatórias de ressecção de segmento ósseo mandibular devido à destruição óssea em decorrência de lesão patológica osteolítica do tipo ameloblastoma

A) Imagem tomográfica tridimensional evidenciando lesão hipodensa na região anterior de mandíbula; B) Imagem do template sobre o biomodelo utilizado para modelar e adaptar a placa de reconstrução; C) Imagem transoperatória evidenciando placa adaptada e fixada previamente à remoção da área tumoral; D) Imagem clínica pós-operatória imediata após a remoção do tumor de origem odontogênica.

Fonte: elaboração dos autores.

\section{Caso clínico 3}

Paciente do gênero feminino, raça branca, 42 anos de idade, encaminhada ao serviço de Cirurgia e Traumatologia Bucomaxilofacial do Hospital Bom Pastor, em Igrejinha, RS, devido à presença de aumento de volume em face ao lado esquerdo e deslocamento da prótese total superior (Figura 3-A). Ao exame clínico intrabucal, observou-se aumento de volume em maxila ao lado esquerdo, com limites desde a linha média anterior até a região posterior da tuberosidade esquerda (Figura 3-B). O exame de imagem evidenciou imagem hipodensa com limite anterior, desde a linha média da maxila, limite posterior sendo a fossa ptérigo-maxilar (Figura 3-C) e limite superior até o bordo do forame infraorbitário. A realização de uma biópsia incisional evidenciou conteúdo sólido no interior da lesão e diagnóstico histológico de Tumor Epitelial Calcificante (Tumor de Pindborg).
O planejamento cirúrgico envolveu uma etapa em que, virtualmente, o tumor foi removido com margem de segurança de $5 \mathrm{~mm}$ em todos os seus limites e também foi confeccionada uma guia cirúrgica mucossuportada para colocação de dois implantes dentários em maxila à direita e na região do malar esquerdo (Figura 3-D). Após a colocação dos implantes por meio de cirurgia guiada à direita em maxila, foi realizada a ressecção do tumor e, posteriormente, fixado um terceiro implante na região de corpo do malar à esquerda, a partir de uma guia ósseossuportada. Sobre biomodelo impresso já sem o tumor (confeccionado a partir da cirurgia virtual), a prótese da paciente foi adaptada por meio de acréscimo de resina acrílica autopolimerizável (Figura 4-A e B), de modo a permitir a captura dos três implantes com o sistema de O'rings ainda no transoperatório (Figura 4-C). 

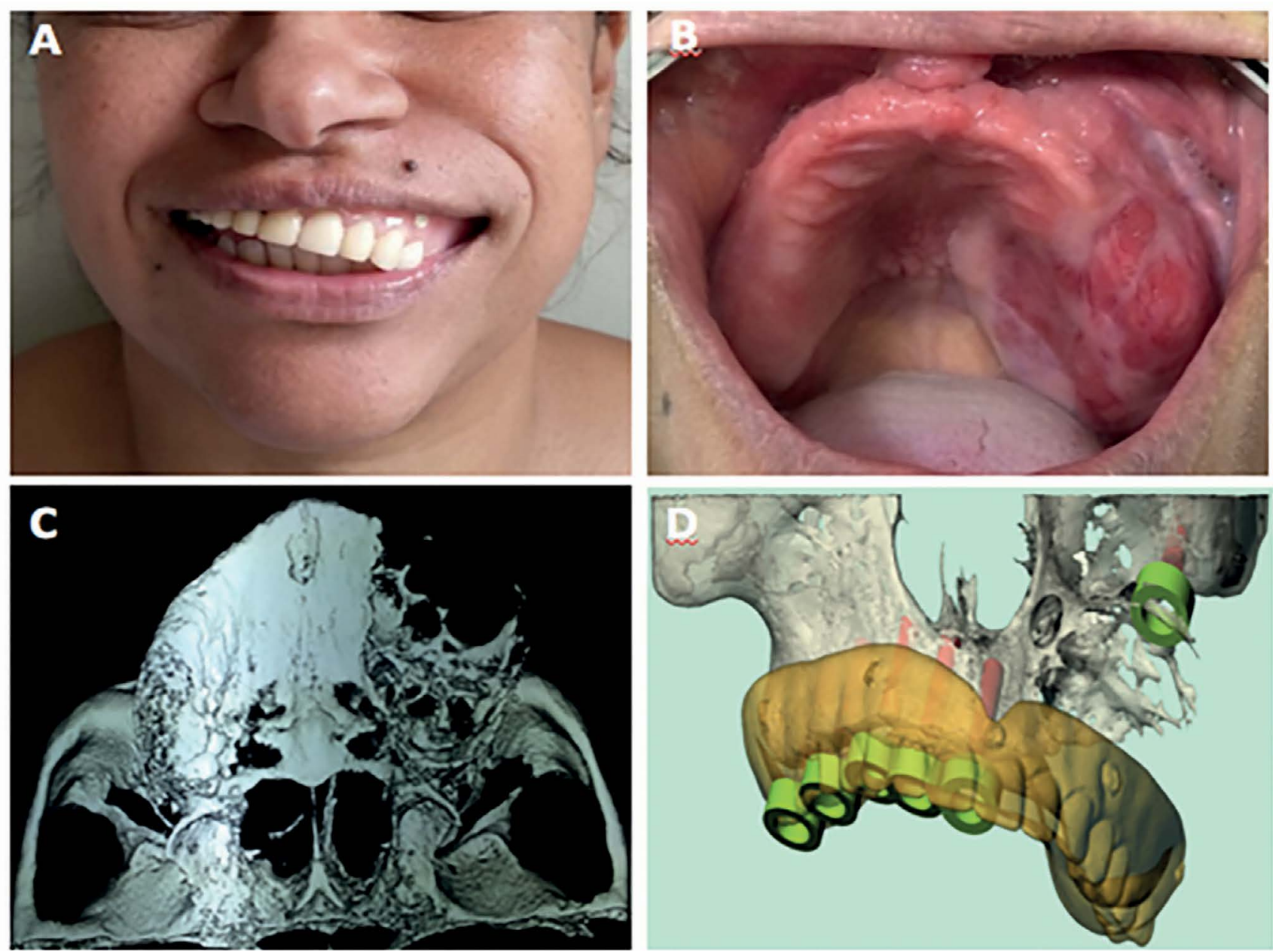

Figura 3 - Imagens pré-operatórias de ressecção de Tumor Epitelial Calcificante (Tumor de Pindborg) em maxila à esquerda

A) Imagem clínica facial evidenciando aumento de volume à esquerda e deslocamento da prótese; B) Imagem clínica intrabucal evidenciando aumento de volume à esquerda em maxila; C) Imagem tomográfica em 3D evidenciando imagem hipodensa à esquerda em maxila; D) Imagem do planejamento virtual evidenciando guia virtual e marcação de locais para inserção dos implantes em maxila à direita e em malar à esquerda.

Fonte: elaboração dos autores.

$\mathrm{O}$ uso do planejamento virtual associado à cirurgia virtual permitiu que, por intermédio do biomodelo já operado, a paciente pudesse sair já da cirurgia com a sua prótese (Figura 4-D). Esse planejamento permitiu um acesso por meio de incisão intrabucal, com colocação imediata de implantes e fixação imediata da prótese como forma de veda- mento do defeito ósseo em maxila e seio maxilar devido à remoção do tumor. A utilização da técnica baseada no uso de biomodelos permitiu menor morbidade pós-operatória da paciente e reinserção social e funcional mais rapidamente quando comparado às técnicas cirúrgicas convencionais. 

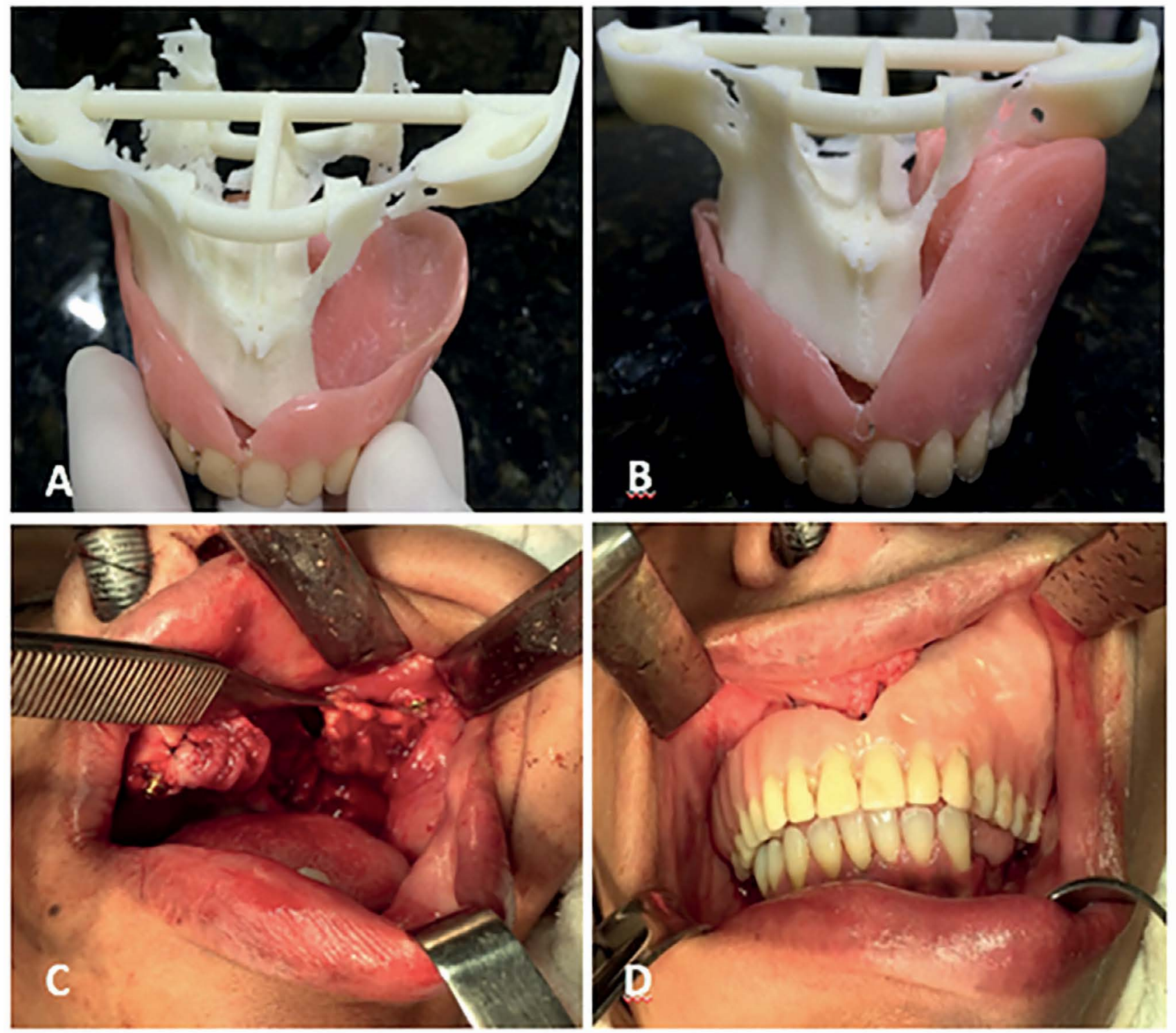

Figura 4 - Imagens pré-operatórias e transoperatórias de ressecção de Tumor Epitelial Calcificante (Tumor de Pindborg) em maxila à esquerda

A) Imagem clínica do biomodelo operado virtualmente em computador e impresso após a cirurgia virtual com a prótese da paciente sem ajustes; B) Imagem clínica do biomodelo operado virtualmente em computador e impresso após a cirurgia virtual com a prótese da paciente já ajustada para ser usada durante a cirurgia; C) Imagem transoperatória evidenciando o defeito ósseo após a remoção da lesão; D) Imagem transoperatória evidenciando a prótese da paciente adaptada e apoiada sobre implantes de modo imediato.

Fonte: elaboração dos autores.

\section{Discussão}

O estudo teve como objetivo mostrar três diferentes aplicações de prototipagens em odontologia por meio de relatos de casos, no qual foram analisadas suas vantagens e desvantagens para cada técnica proposta e executada. A utilização de biomodelos, como visto nos três casos que foram ilustrados, permitiu que a intervenção técnica fosse realizada por meio de acesso cirúrgico intraoral, sendo, portanto, muito mais conservadora e estética. Isso permitiu ao paciente a ausência de cicatriz em face e a diminuição da morbidade pós-operatória, de recidivas e de danos às estruturas nobres (nervos e vasos), fato que corrobora os dados da literatura ${ }^{5,10}$. Essas vantagens também podem ser encontradas em outros estudos, como o de Wang ${ }^{8}$, no qual foi realizado um planejamento com biomodelos para cirurgia pediátrica com enorme redução da morbidade pós-operatória.

A utilização das técnicas que fazem o uso de biomodelos não fica somente restrita à área odontológica, sendo também muito utilizada em cirurgias de crânio, como mostra o estudo de $\operatorname{Sun}^{6}$, em que quatro pacientes foram submetidos à remodelação da abóbada craniana e foi realizado o planejamento virtual e sua construção em CAD/CAM, sendo que essas guias melhoraram a precisão operatória e aceleraram o transoperatório, além de facilitar a visualização pré-operatória e a compreensão dos pacientes e seus familiares com o auxílio da cirurgia virtual.

A manutenção relativa do contorno facial e do posicionamento fisiológico das articulações tempo- 
romandibulares (ATM) é algo que a utilização dos biomodelos, em cirurgias bucomaxilofaciais, apresenta como fator positivo. Com a manutenção em posição fisiológica adequada dos côndilos com a fossa glenoide, há a redução de riscos da ocorrência de problemas de ATM no pós-operatório. Segundo Ciocca $^{7}$, após um acompanhamento de cinco anos de pacientes que realizaram cirurgia de côndilo com 0 auxílio de prototipagens em CAD/CAM, melhorando a precisão anatômica, melhora-se a dinâmica dos tecidos articulares, sendo que o uso de biomodelos favorece a obtenção de melhores resultados.

A redução do tempo operatório é notória, uma vez que a utilização de biomodelos torna o planejamento e a execução cirúrgica mais precisos e menos complexos quando comparados às técnicas cirúrgicas convencionais executadas sem o uso de biomodelos. Consequentemente, a redução da morbidade pós-operatória ocorre de forma paralela à redução da complexidade da execução da cirurgia, conforme observado também em outros trabalhos ${ }^{1,8,10}$ que embasam o uso de biomodelos, como nos casos ilustrados neste trabalho. Contudo, o custo financeiro da utilização dos biomodelos é visto como uma desvantagem $^{5}$, o que, na visão de outros autores, não se torna um custo elevado frente aos benefícios que 0 uso do biomodelo pode trazer ao paciente.

Portanto, o uso de biomodelos em odontologia, mais especificamente em cirurgia e traumatologia bucomaxilofacial, ainda é uma técnica relativamente pouco difundida, mas muito promissora, uma vez que leva ao profissional a aplicação de técnicas cirúrgicas mais previsíveis e, ao paciente, menor morbidade pós-operatória.

\section{Abstract}

Objective: to present the possibilities for using prototyping (biomodels) in Oral and Maxillofacial Surgery and Traumatology through the reporting of clinical cases. Case reports: the present study covers three clinical situations. The first one is a patient with osteoradionecrosis associated with pathological fracture due to an evolution of osteolysis condition, whereas fragment resection was performed, as well as the adaptation of the reconstruction plate on the biomodel. The second case reports the presence of a Multicystic Ameloblastoma covering symphysis, parasymphysis, and mandible body, and prototyping and adaptation of the reconstruction surgical plate were used. The last report was of a patient diagnosed with Calcifying Epithelial Tumor from the midline of the maxilla to the left pterygoid-maxillary fossa. The use of biomodels allowed producing an implant-supported complete denture that would seal the bone defect. Final considerations: it is notable that the use of biomodels aid surgical planning and execution, expanding treatment alternatives that enable the rehabilitation of the patient with more effective surgical techniques, less morbidity, and less surgical time.

Keywords: Three-dimensional priting. Oral surgery. Pathology.

\section{Referências}

1. Shankaran G, Deogade SC, Dhirawani R. Fabrication of a Cranial Prosthesis Combined with an Ocular Prosthesis Using Rapid Prototyping: a case report. J Dent (Tehran) 2016; 13(1):68-72.

2. Cassetta M, Giansanti M, Di Mambro A, Calasso S, Barbato E. Minimally invasive corticotomy in orthodontics using a three-dimensional printed $\mathrm{CAD} / \mathrm{CAM}$ surgical guide. Int $\mathrm{J}$ Oral Maxillofac Surg 2016; 45(9):1059-64.

3. Kumta S, Kumta M, Jain L, Purohit S, Ummul R. A novel 3D template for mandible and maxilla reconstruction: Rapid prototyping using stereolithography. Indian J Plast Surg 2015; 48(3):263-73.

4. Choi JY, Choi JH, Kim NK, Kim Y, Lee JK, Kim MK, et al. Analysis of errors in medical rapid prototyping models. Int $\mathrm{J}$ Oral Maxillofac Surg 2002; 31(1):23-32.

5. Torabi K, Farjood E, Hamedani S. Rapid Prototyping Technologies and their Applications in Prosthodontics, a Review of Literature. J Dent (Shiraz) 2015; 16(1):1-9.

6. Sun H, Li B, Zhao Z, Zhang L, Shen SG, Wang X. Error analysis of a CAD/CAM method for unidirectional mandibular distraction osteogenesis in the treatment of hemifacial microsomia. Br J Oral Maxillofac Surg 2013; 51(8):892-7.

7. Ciocca L, Tarsitano A, Marchetti C, Scotti R. A CAD-CAM-prototyped temporomandibular condyle connected to a bony plate to support a free fibula flap in patients undergoing mandiblectomy: A pilot study with 5 years of follow up. J Craniomaxillofac Surg 2016; 44(7):811-9.

8. Wang C, Chen Y, You Y, Wang M, Lv C, Gui L. Surgical Template to Minimize the Damage of Tooth Buds in Young Children With Mandibular Distraction Osteogenesis. J Craniofac Surg 2016; 27(7):1732-4.

9. Nayar S, Bhuminathan S, Bhat WM. Rapid prototyping and stereolithography in dentistry. J Pharm Bioallied Sci 2015; 7(Suppl 1):S216-9.

10. Fernandes N, van den Heever J, Hoogendijk C, Botha S, Booysen G, Els J. Reconstruction of an Extensive Midfacial Defect Using Additive Manufacturing Techniques. J Prosthodont 2016; 25(7):589-94.

\section{Endereço para correspondência:}

Thiago Calcagnotto

Rua São João, 942 Centro

93010-250 São Leopoldo - RS

Fone: (51) 991746104

E-mail: tcalcagnotto@hotmail.com

Recebido: 06/01/2017. Aceito: 03/04/2017. 